\title{
A triangle model of criminality
}

\author{
Juan C. Nuño , Miguel A. Herrero , Mario Primicerio \\ Departamento de Matemática Aplicada a los Recursos Naturales, E.T.S.I. Montes, Universidad Politécnica de Madrid, 28040 Madrid, Spain \\ Departamento de Matemática Aplicada, Facultad de Ciencias Matemáticas, Universidad Complutense de Madrid, 28040 Madrid, Spain \\ Dipartimento di Matematica "Ulisse Dini", Università degli Studi di Firenze, 55015 Firenze, Italy
}

\begin{abstract}
This paper is concerned with a quantitative model describing the interaction of three sociological species, termed as owners, criminals and security guards, and denoted by $X, Y$ and $Z$ respectively. In our model, $Y$ is a predator of the species $X$, and so is $Z$ with respect to $Y$. Moreover, $Z$ can also be thought of as a predator of $X$, since this last population is required to bear the costs of maintaining $Z$.

We propose a system of three ordinary differential equations to account for the time evolution of $X(t), Y(t)$ and $Z(t)$ according to our previous assumptions. Out of the various parameters that appear in that system, we select two of them, denoted by $H$, and $h$, which are related with the efficiency of the security forces as a control parameter in our discussion. To begin with, we consider the case of large and constant owners population, which allows us to reduce (3)-(5) to a bidimensional system for $Y(t)$ and $Z(t)$. As a preliminary step, this situation is first discussed under the additional assumption that $Y(t)+Z(t)$ is constant. A bifurcation study is then performed in terms of $H$ and $h$, which shows the key role played by the rate of casualties in $Y$ and $Z$, that results particularly in a possible onset of bistability. When the previous restriction is dropped, we observe the appearance of oscillatory behaviours in the full two-dimensional system. We finally provide a exploratory study of the complete model (3)-(5), where a number of bifurcations appear as parameter $H$ changes, and the corresponding solutions behaviours are described.
\end{abstract}

Keywords: Criminality; Nonlinear dynamics; Sociological systems

\section{Introduction}

Crime has always been a serious concern in human societies. Indeed, references to criminal behaviour (and measures to check it) are well documented from the oldest extant records to the present (cf. Refs. $[1,2,25,3,4]$ ). While the concept of criminal (hence unacceptable) conduct largely differs among various cultures and historical periods, there is some general agreement in considering certain acts as criminal. In particular, this applies to the unauthorized 
exaction of resources belonging to a person or group of persons, legally considered to be their owners. This is the type of criminal behaviour addressed in this paper.

It is widely assumed that there is an enormous variability in crime rates, both in space and time [26]. Herein, we shall be concerned with some quantitative models that describe the evolution in time of three interacting populations. They will be referred to as owners, criminals and security guards, respectively. These are the three essential players in the so called Routine Activity Theory , one of the current conceptual frameworks being used in quantitative criminology

. As concerns the interaction of these species, the following assumptions will be made:

A.1 Owners population (to be denoted as $X(t)$ ) are prey to the criminal population (to be denoted as $Y(t)$ ).

A.2 Security guard population (to be represented as $Z(t)$ ) not only acts as predator on the criminal population, but also on the owners', since the latter has to bear the cost of keeping the actual numbers of $Z(t)$.

A.3 A crime is committed whenever a criminal meets an owner in the absence of security surveillance. This statement is often referred to as the Triangle Dogma

A.4 In any criminal event, two characteristic time scales can be distinguished. More precisely, a first period in which a criminal stalks prey is followed by a second stage in which that criminal is actively and exclusively engaged in committing that crime. Assuming this behaviour, the functional response of the criminal population $C_{f}(t)$ is given by Refs.

$$
C_{f}(t)=\frac{k X(t)}{E+X(t)} Y(t)
$$

for some constants $k \geq 0$ and $E \geq 0$.

A.5 The process of neutralisation of criminals by security forces follows a kinetics similar to that described in our previous hypothesis. More precisely, the rate of removal of criminals by the guards actions, $S_{f}(t)$, is given by:

$$
S_{f}(t)=\frac{H Y(t)}{D+Y(t)} Z(t)
$$

for some $H \geq 0$ and $D \geq 0$. The parameter $H$ will be termed as the efficiency of the security guards and will play a key role in the forthcoming study.

We next state the triangle model which incorporates assumptions (A.1)-(A.5). It reads as follows:

$$
\begin{aligned}
\frac{\mathrm{d} X}{\mathrm{~d} t} & =r(N-X)(K-X)-\frac{k X}{E+X} Y-B \frac{Z}{X} \\
\frac{\mathrm{d} Y}{\mathrm{~d} t} & =f \frac{S X}{E+X} Y-\frac{H Y}{D+Y} Z-F Y-G Y^{2} \\
\frac{\mathrm{d} Z}{\mathrm{~d} t} & =g \frac{S X}{E+X} Y-h \frac{H Y}{D+Y} Z-C Z .
\end{aligned}
$$

Concerning Eqs. (3)-(5), some remarks are in order. To begin with, space-dependent properties are not included in this system. Moreover, (3)-(5) represent a continuum-based deterministic approach. This framework is particularly well suited to detecting changes in the dynamics of solutions associated with bifurcations arising when some control parameter reaches critical values, a strategy that will be exploited in the sequel. Discrete and stochastic effects are therefore excluded (or averaged out in a suitable manner). For a general discussion on the features of these different modelling approaches, the reader is referred to Ref.

Eq. (3) asserts that, in the absence of criminals and guards, the owners population obeys a logistic-type dynamics with a growth rate $r>0$ and a maximum capacity $N>0$. The parameter $K<N$ is the optimal reachable population and so, $X(t) \rightarrow K$ as $t \rightarrow \infty$ if either $0 \leq X \leq K$ or $K \leq X<N$. Note that in this formulation the extinction state $X=0$ is not a steady state. The presence of criminals hinders the growth of $X$ at a rate given by (1), as described by the second term on the right hand side of (3). In its turn, the existence of security forces represents a cost which is shared by all owners in the manner described by the latest term on the right hand side of (3), with $B>0$ there.

Eq. (4) describes the evolution in time of the criminal population $Y(t)$. It is therein assumed that $Y(t)$ increases according to the criminal rate (1) with a proportionality constant $f>0$. On the other hand, $Y(t)$ decreases under the 
action of $Z(t)$ (recall (2)) as a consequence of interspecific competition. This last action is represented by the third and fourth terms on the right hand side of (4).

Finally, the security forces growth is proportional (with a rate constant $g>0$ ) to the crimes committed, as shown by the first term on the right hand side of (5). On the other hand, $Z(t)$ decreases as a consequence of the casualties suffered in encounters with the criminals, the corresponding rate constant being $h>0$. We point out that the concept of casualty in this context is to be associated with the loss of function in general. More precisely, it includes deactivation of security forces by corruption and other mechanisms, as for instance by moving police forces out of a particularly difficult neighbourhood. Natural decay (with constant $C>0$ ) also has a negative impact on the values of $Z(t)$.

We should stress that, in order to assess the actual relevance of models like (3)-(5), reliable estimates for constants $k, E, B, H, f, g, h, G$ and $C$ should be provided by sociological records. Obtaining such values seems to be a major challenge for the mathematical modelling in the field. This last approach is not entirely new

but is not universally accepted either $\quad$. However, in recent years a number of works have addressed the issue of studying the evolution in time of a population where criminals are present by means of a mathematical analysis . A similar point of view has been used to describe dynastic cycles

We conclude this introduction by summarising the plan of this note. We first consider in Section 2 below the case of constant (and large) owners population. This assumption allows us to reduce (3)-(5) to a bidimensional system that can be analysed by means of phase space techniques. Under the additional assumption that $(Y+Z)$ remains constant for all times, we are able to discuss in detail the dynamic behaviour of solutions in terms of $\mathrm{H}$ for a given set of parameter values. In particular, the possibility of severe depletion in the active population of security guards is shown to occur whenever $h>0$. When the previous assumption on $(Y+Z)$ is dropped, an oscillatory dynamic appears, which is discussed in the last paragraph of that section. We then consider in Section 3 the case of the full system (3)-(5). The corresponding dynamics are extremely rich, and we have reduced ourselves to providing a quick discussion on the influence of parameter $\mathrm{H}$ in the behaviour of solutions. To this end, a reference parameter set is selected. Regardless of the limited character of that choice, a number of bifurcations are identified, and the corresponding dynamics are succinctly described. Finally some concluding remarks are gathered in Section 4 at the end of this article.

\section{A reduced system: The case of a constant and large owners population}

In many practical circumstances, the number of owners that are potential targets for criminals can be assumed to be constant and large with respect to the corresponding figures for criminals and guardians. In other words,

$$
X(t) \equiv X_{0} \gg Y(t)+Z(t) \quad \text { for all } t>0 .
$$

Under this assumption, the hypothesis $X(t)=X_{0}$ can be assumed to hold for sufficiently large times, and (4) and (5) reduces to

$$
\begin{aligned}
& \frac{\mathrm{d} Y}{\mathrm{~d} t}=f \alpha Y-Y(F+G Y)-\frac{H Y}{D+Y} Z \\
& \frac{\mathrm{d} Z}{\mathrm{~d} t}=g \alpha Y-h \frac{H Y}{D+Y} Z-C Z
\end{aligned}
$$

where

$$
\alpha=\frac{k X_{0}}{E+X_{0}} .
$$

A further simplification is achieved when the system is subject to global regulation as described below.

\subsection{A bidimensional system with competition}

To begin with, we consider a simplified case. More precisely, we further introduce the assumption that the system has a first integral, namely:

$$
Y(t)+Z(t)=M \quad \text { for all } t>0 .
$$


While the hypothesis might be arguable in itself, we believe that the reduced system thus obtained still deserves some consideration. Indeed, as noted below, the role of the parameter $h$ will be clearly highlighted in this case. Moreover, conditions akin to (9) are commonly used in ecology, at least at a preliminary modelling level. For instance, the existence of a first integral is the key to derive the classical oscillatory Lotka-Volterra dynamics . Furthermore, an assumption similar to (9) is customarily made when dealing with the quasispecies theory in prebiotic evolution

A neutral and natural way of ensuring this constraint is by introducing a negative flux $\phi=\phi(Y, Z)$ in Eqs. (7) and (8). Thus, (7) and (8) are replaced by the following modified system:

$$
\begin{aligned}
\frac{\mathrm{d} Y}{\mathrm{~d} t} & =f \alpha Y-Y(F+G Y)-\frac{H Y}{D+Y} Z-\phi Y \\
\frac{\mathrm{d} Z}{\mathrm{~d} t} & =g \alpha Y-C Z-h \frac{H Y}{D+Y} Z-\phi Z .
\end{aligned}
$$

Furthermore, by absorbing linear terms in $\phi$ we may take $F=C=0$ without loss of generality. At this stage, it is convenient to introduce nondimensional variables by setting:

$$
y=\frac{Y}{M} ; \quad z=\frac{Z}{M} ; \quad d=\frac{D}{M} ; \quad \xi=M G .
$$

One then has that (9) yields

$$
z=1-y
$$

and (10) and (11) transform into

$$
\begin{aligned}
& \frac{\mathrm{d} y}{\mathrm{~d} t}=f \alpha y-\xi y^{2}-\frac{H y(1-y)}{d+y}-\phi y \\
& \frac{\mathrm{d} z}{\mathrm{~d} t}=g \alpha y-h \frac{H y(1-y)}{d+y}-\phi(1-y) .
\end{aligned}
$$

On imposing (13), we readily obtain from (14) and (15) that

$$
\phi(y)=(f+g) \alpha y-\xi y^{2}-(1+h) H \frac{y(1-y)}{d+y}
$$

so that $y(t)$ satisfies:

$$
\frac{\mathrm{d} y}{\mathrm{~d} t}=y\left((1-y)\left(f \alpha-\xi y-\frac{H}{\mathrm{~d}+y}+H(1+h) \frac{y}{\mathrm{~d}+y}\right)-g \alpha y\right) .
$$

Eq. (17) does not seem to be analytically solvable. However, the behaviour of its solutions can be qualitatively discussed in terms of the parameters appearing therein. We shall select $H$ (the police efficiency, cf. (2)) as our main control parameter, and in the sequel we shall describe the various behaviours that appear as $H$ varies, with the remaining constants kept fixed. In particular, let us take:

$$
\alpha=10^{2} ; \quad d=10^{-1} ; \quad g=10^{-2} ; \quad f=10^{-1} ; \quad h=10^{-1} ; \quad \xi=1.0 .
$$

While no claim is made herein on the actual relevance of (18) in any particular criminal setting, some remarks can be made on the rationale behind such a choice. For instance, in view of (1) the (comparatively) large value of $\alpha$ in (18) corresponds to a large value of $X_{0}$ and $k$. A similar argument (replacing $X_{0}$ by $M$ ) also applies to the values of $d$ and $\xi$ in (18). On the other hand, the fact that $0<g<f$ and that they differ by one order of magnitude, corresponds to the assumption that both criminal and security guard numbers increase in proportion to crimes committed, but the second population does so at a slower pace than the first one. Finally, the choice $h=10^{-1}$ (which shall be reconsidered later) corresponds to a relatively high casualty rate for guards when interacting with criminals. 


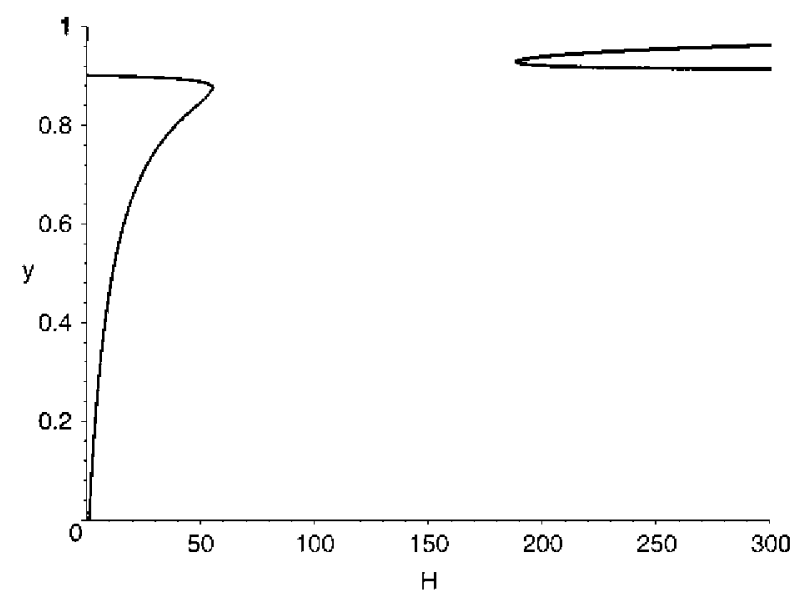

Fig. 1. Bifurcation diagram for the 2D-competition model as a function of $H$.

We proceed next to discuss the behaviour of solutions to (17). To this end, we first observe that the equation has a trivial steady state

$$
\bar{y}_{0}=0 ;
$$

which is independent of the parameter choice in (17). This value means extinction of the criminal population. Note, however, that the extinction of the guards is not a steady state for (17). The remaining steady states are given by the roots of the cubic equation:

$$
\begin{aligned}
\xi y^{3} & +(\xi(d-1)-(f+g) \alpha-H(1+h)) y^{2} \\
& +(f \alpha(1-d)-\xi d+H(2+h)-g \alpha d) y+f \alpha d-H=0 .
\end{aligned}
$$

While solutions of (19) can be represented by Cardano-Viete's formulae (cf, for instance, Ref. [19]), the expressions thus obtained are not particularly illuminating. Actually, since Eq. (19) is linear in $h$, we can write $H$ in terms of $y$ in the form $H(y)=\frac{f(y)}{g(y)}$, where $f$ and $g$ are cubic and quadratic polynomials in $y$, respectively. Under our current choice of parameters, one of these roots is always real and larger than one for all values of $H \geq 0$, so it is not relevant for our discussion here. The remaining two roots $y_{1}, y_{2}$ are both real and satisfy $0<y_{1}<y_{2}<1$ for some values of $H$, namely

$$
0<H<55.25 \equiv H_{1} \quad \text { and } \quad H>188.75 \equiv H_{2} .
$$

Thus, two branches of $y$ exist for $0<H<H_{1}$ or $H>H_{2}$. Moreover, for $0<H<1 \equiv H_{c}$ the lower branch is negative. It is important to observe that the value $H_{C}=1$ remains constant when $h$ is varied. This situation is depicted in Fig. 1.

Using standard bifurcation techniques , it is straightforward to show that:

- $y_{0}$ is asymptotically stable for $H_{C}<H$.

- $y_{1}>0$ (respectively $y_{2}>0$ with $0<y_{1}<y_{2}<1$ ) is asymptotically stable (respectively unstable) in the range $H_{c}<H<H_{1}$ and $H>H_{2}$.

The global shape of this bifurcation diagram depends crucially on the value of the casualty parameter $h$. As a matter of fact, one has that:

As $h \rightarrow 0$, then $H_{1} \rightarrow 23.64$ and $H_{2} \rightarrow \infty$, whenever the remaining values in (18) are kept fixed.

A striking consequence of (A)-(B) is that for $h=0$ only, an increase in security efficiency (so that $H>H_{1}$ ) guarantees the effective removal of criminals from the system. On the other hand, whenever $h>0$, and no matter how small $h$ is, a criminal population could remain active for arbitrarily large values of $H$ (particularly for some initial conditions).

A further point to be noted is that a global bifurcation appears for $h=h_{c} \equiv 0.1099019514$, which leads to the onset of bistability. Specifically, the upper branch in the left and the lower branch in the right of Fig. 1 coalesce when 


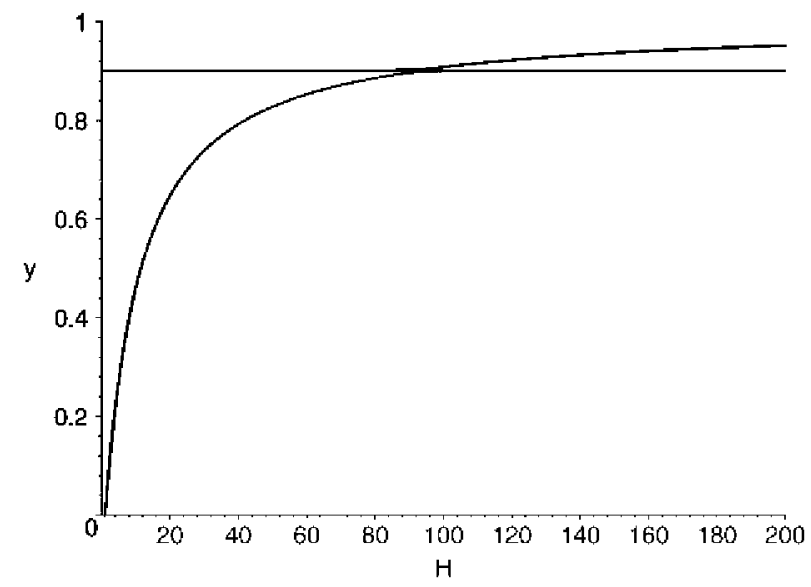

Fig. 2. Coalescence of the two branches of steady states at $h \equiv h_{C}$.

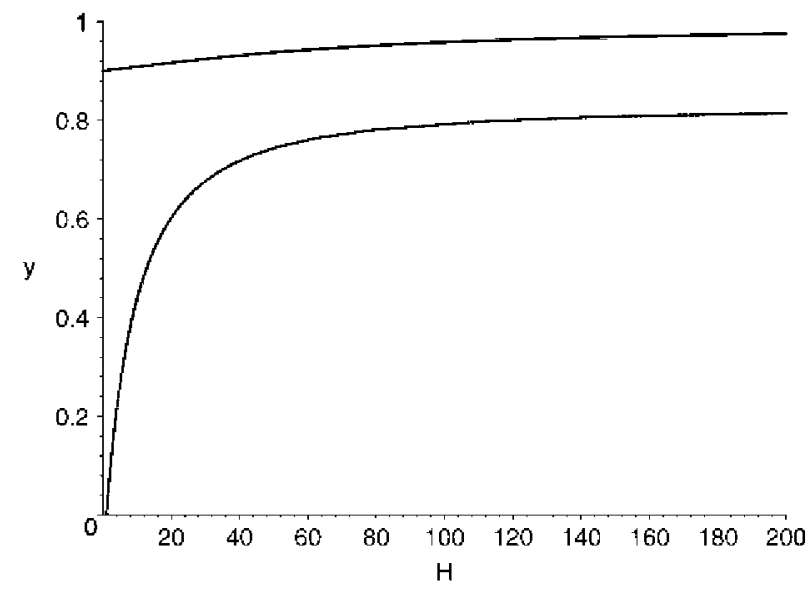

Fig. 3. The bifurcation diagram for $h>h_{c}$.

$h=h_{c}$. When $h>h_{c}$, i.e. when losses in the guardian population due to the fight against criminals are relevant, even a huge increase in police efficiency is not enough to get rid of criminality. This situation is summarised in Figs. 2 and 3.

\subsection{The model without regulation: Oscillatory behaviours}

When condition (9) is dispensed with, the dynamics of (7) and (8) becomes more complex. To compare this with the case considered in our previous paragraph, we now select parameters as follows:

$$
\begin{aligned}
& \alpha=10^{2} ; \quad d=10^{-1} ; \quad g=10^{-2} ; \quad f=10^{-1} ; \\
& h=0 ; \quad C=1 ; \quad F=10^{-1} ; \quad G=1 .
\end{aligned}
$$

Under such assumptions (7) and (8) has two nonnegative steady states given by $P_{1}(0,0)$ and $P_{2}(\bar{y}, \bar{y})$, where

$$
\bar{y}=4.9-\frac{H}{2}+\frac{1}{10} \sqrt{2500-490 H+25 H^{2}} .
$$

The equilibrium $P_{2}$ corresponds to the coexistence of criminals and security forces. As $H$ increases, $P_{2}$ moves towards the origin, so that higher security efficiency leads to the extinction of both populations. A stability analysis of $P_{2}$ in terms of $H$ reveals a number of bifurcations, including in particular two Andronov-Hopf bifurcation types at the values $\bar{H}_{2}$ and $\bar{H}_{5}$ described in Table 1 . 
Table 1

Classification of $P_{2}$ for different ranges of $H$

\begin{tabular}{lc}
\hline$H$ & Type of equilibria \\
\hline$\left(0, \bar{H}_{1}\right)$ & STABLE NODE \\
$\left(\bar{H}_{1}, \bar{H}_{2}\right)$ & STABLE FOCUS \\
$\bar{H}_{2}$ & CENTER \\
$\left(\bar{H}_{2}, \bar{H}_{3}\right)$ & UNSTABLE FOCUS \\
$\left(\bar{H}_{3}, \bar{H}_{4}\right)$ & UNSTABLE NODE \\
$\left(\bar{H}_{4}, \bar{H}_{5}\right)$ & UNSTABLE FOCUS \\
$\bar{H}_{5}$ & CENTER \\
$\left(\bar{H}_{5}, \infty\right)$ & STABLE FOCUS \\
\hline
\end{tabular}

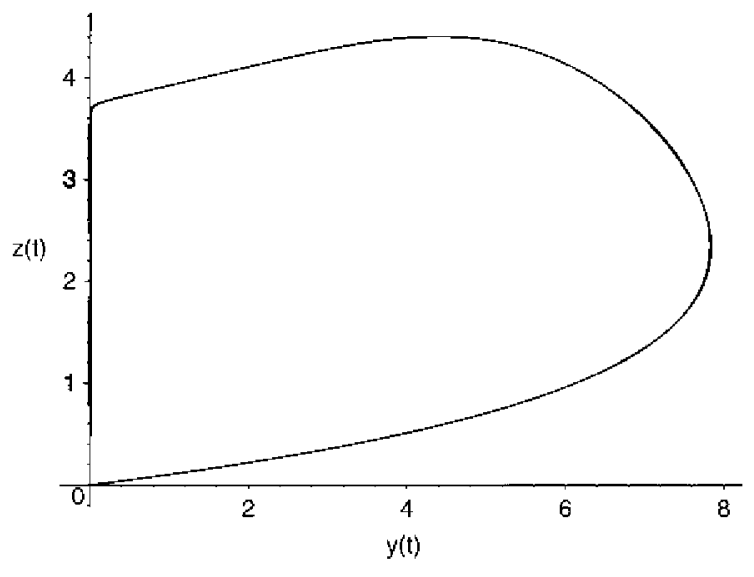

Fig. 4. Limit cycle for (7) and (8) when (22) holds and $H=7$.

The critical values of $H$ are approximately given by:

$$
\bar{H}_{1} \approx 0.28 ; \quad \bar{H}_{2} \approx 5.64 ; \quad \bar{H}_{3} \approx 7.56 ; \quad \bar{H}_{4} \approx 17.785 ; \quad \bar{H}_{5} \approx 96.65 .
$$

As an illustration of the behaviours obtained, some features of the case $H=7$ are depicted in Figs. 4-6. Fig. 4 shows a plot of the limit cycle that appears around the equilibrium point $P_{2}$, which corresponds to the unstable coexistence of both species. As observed before, $P_{2}$ moves towards the origin as $H$ increases. A close-up of the evolution in time of $y(t)$ and $z(t)$ is provided in Fig. 5. As depicted therein, two time scales can be distinguished in the evolution of the criminal population. In particular, a sudden increase in $y(t)$ occurs for low values of $z(t)$, which quickly grows to larger values. This is followed by a long period of stable, low criminality values. In turn, the security forces then relax their members to match this new situation, although at a slower pace. This general trend is preserved as $H$ varies. Finally, a general picture of $(y(t), z(t))$ along several oscillations in the limit cycle is given in Fig. 6 .

Note that the response of the security guards to the criminal challenge is one of a quick fight and a slow adjustment to a scenario of low criminality. A remarkable feature is the fast control of the system by criminals under favourable circumstances. Note also that, as shown in Fig. 6, the system remains for most of the time in a situation of low criminality, i.e. of low values for $y(t)$. Under this situation security forces are not required, so that values of $z(t)$ remain also small.

\section{Some remarks on the complete triangle model}

In this section, we provide a short description of the bifurcations that appear in the complete 3-D system (3)-(5) in terms of the parameter $H$, when the remaining parameters are kept fixed to a reference value (cf. (27)) given below. To this end, we introduce nondimensional variables as follows:

$$
\tau=r N t ; \quad x=\frac{X}{N} ; \quad y=\frac{Y}{N} ; \quad z=\frac{Z}{N} .
$$




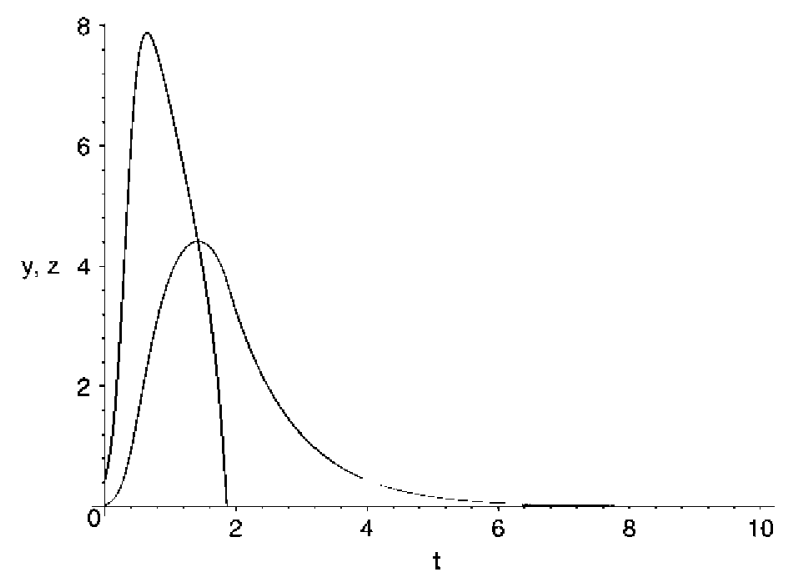

Fig. 5. The profiles of $y(t)$ and $z(t)$ during one period in the previous case.

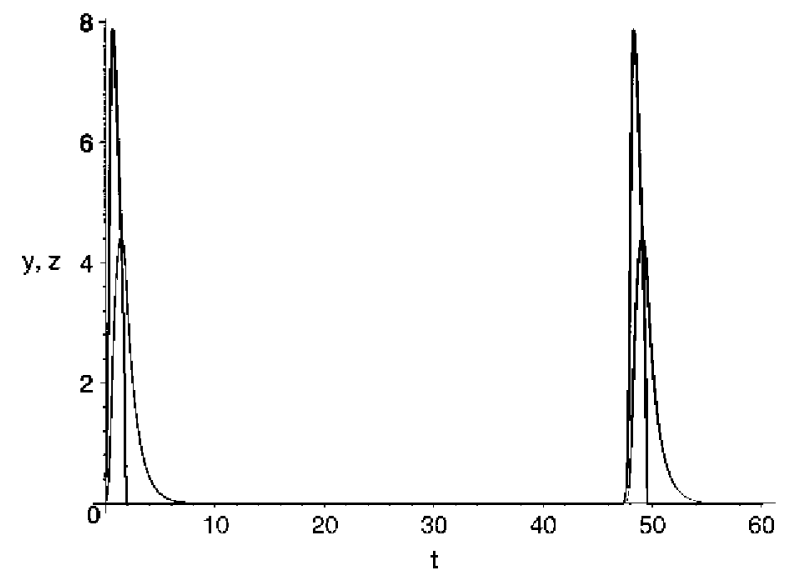

Fig. 6. Slow and fast relaxations corresponding to the limit cycle of Fig. 4.

And new parameters given by

$$
\begin{aligned}
& \xi=\frac{k}{r N} ; \quad \beta=\frac{B}{r N^{2}} ; \quad \epsilon=\frac{E}{N} ; \quad H_{1}=\frac{H}{r N} ; \quad d=\frac{D}{N} \\
& \rho=\frac{F}{r N} ; \quad \gamma=\frac{C}{r N} ; \quad \mu=\frac{G}{r} ; \quad \kappa=\frac{K}{N} .
\end{aligned}
$$

Then, on replacing $H$ by $H_{1}=\frac{H}{r N}$ and dropping (for simplicity) the subscript in $H_{1}$, (3)-(5) is recast in the following nondimensional form:

$$
\begin{aligned}
& \frac{\mathrm{d} x}{\mathrm{~d} \tau}=(1-x)(\kappa-x)-\frac{\xi x}{\epsilon+x} y-\beta \frac{z}{x} \\
& \frac{\mathrm{d} y}{\mathrm{~d} \tau}=f \frac{\xi x}{\epsilon+x} y-\frac{H y}{d+y} z-\rho y-\mu y^{2} \\
& \frac{\mathrm{d} z}{\mathrm{~d} \tau}=g \frac{\xi x}{\epsilon+x} y-h \frac{H y}{d+y} z-\gamma z .
\end{aligned}
$$

To proceed further, we select a reference parameter set that corresponds to the values:

$$
\begin{array}{ll}
\kappa=0.5 ; & \beta=10^{-1} ; \quad \rho=10^{-2} ; \quad \gamma=10^{-1} ; \quad d=10^{-3} ; \quad \xi=4 \cdot 10^{6} ; \quad \epsilon=10^{6} ; \\
f=0.1 ; \quad g=7 ; \quad 10^{-2} ; \quad h=0 ; \quad \mu=0 .
\end{array}
$$


Table 2

A description of the bifurcation dynamics for (25)-(27) in terms of $H$

\begin{tabular}{ll}
\hline$H$ & Type of dynamics \\
\hline$\left(0, \widetilde{H}_{1}\right)$ & GLOBAL FIXED POINT ATTRACTOR \\
$\widetilde{H}_{1}$ & GENERALIZED HOPF BIFURCATION \\
$\left(\widetilde{H}_{1}, \widetilde{H}_{2}\right)$ & BISTABILITY OF CYCLES \\
$\widetilde{H}_{2}$ & SUB-CRITICAL HOPF BIFURCATION \\
$\left(\widetilde{H}_{2}, \widetilde{H}_{3}\right)$ & A SINGLE, ASYMPTOTICALLY STABLE, LIMIT CYCLE \\
$\widetilde{H}_{4}$ & HOPF BIFURCATION \\
$\left(\widetilde{H}_{4}, \infty\right)$ & STABLE FOCUS \\
\hline
\end{tabular}

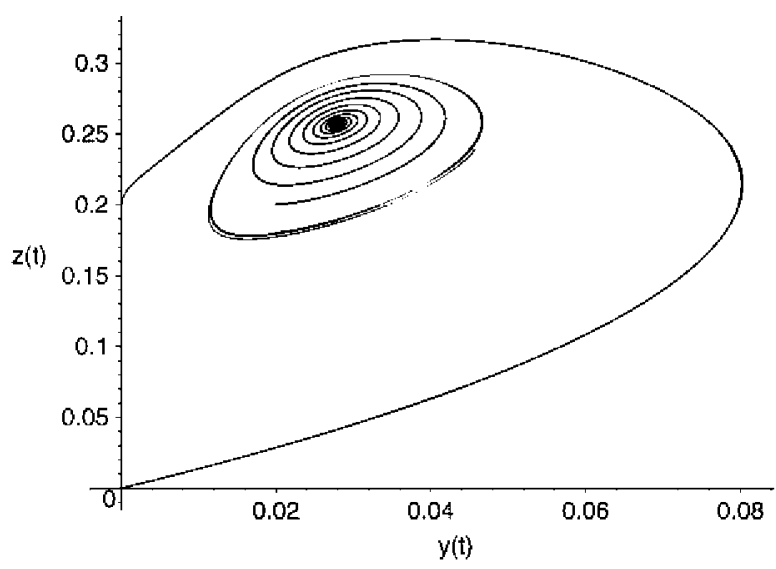

Fig. 7. The projection of the phase space onto the $(y, z)$-plane when $H=0.00355$. Two limit cycles surround the stable fixed point. The external cycle is asymptotically stable, whereas the internal one is unstable.

As $H$ increases from zero to infinity, a bifurcation dynamics appears (see Table 2):

A global bifurcation occurs at the value $H=\widetilde{H}_{1}$, and an unstable limit cycle then appears surrounding a stable steady state. As the parameter $H$ increases, this limit cycle splits into two: an external one (asymptotically stable) and an internal one (unstable). This is an example of a transcritical bifurcation from a family of limit cycles, also termed as a generalized Hopf bifurcation or saddle-node bifurcation of cycles . Estimated values for $\widetilde{H}_{1}, \widetilde{H}_{2}$ and $\widetilde{H}_{3}$ are given by

$$
\widetilde{H}_{1}=0.00324 ; \widetilde{H}_{2}=0.004 ; \widetilde{H}_{3}=0.05 \text {. }
$$

In Fig. 7, we provide a picture obtained by a projection over the $(y, z)$-plane for the choice made in (27) and $H=0.00355$. The corresponding tridimensional plot can be seen in Fig. 8. Such qualitative behaviour is preserved until $H=\widetilde{H}_{2}$, when the internal limit cycle collapses and the equilibrium point becomes unstable. Then a subcritical Hopf bifurcation occurs . When $\mathrm{H}$ moves from $\widetilde{H}_{2}$ to $\widetilde{H}_{3}$, only one (asymptotically stable) limit cycle exists. As $H$ further increases, this limit cycle reduces its size and simultaneously approaches the point $\left(\frac{1}{2}, 0,0\right)$, which corresponds to the extinction of both the criminals and the guards. However, this equilibrium is unstable under the current assumptions. As $H$ reaches $\widetilde{H}_{3}$, the limit cycle disappears and the focus becomes stable again.

\section{Concluding remarks}

The model considered in this paper describes the interaction between two predators (criminals and security guards) which compete for the same resources. These predators are provided a single prey species (the owners). However, the behaviours of these two populations differ sharply from each other. While criminals act as standard predators of the owners, guards do so only by diminishing resources, in a manner proportional to their number, which is shared equally by all members of the owners population. On the other hand, criminals and guards compete among themselves. This results in casualties on both sides, whose relative strengths are measured by parameters $H$ and $h$ in (4) and (5). The 


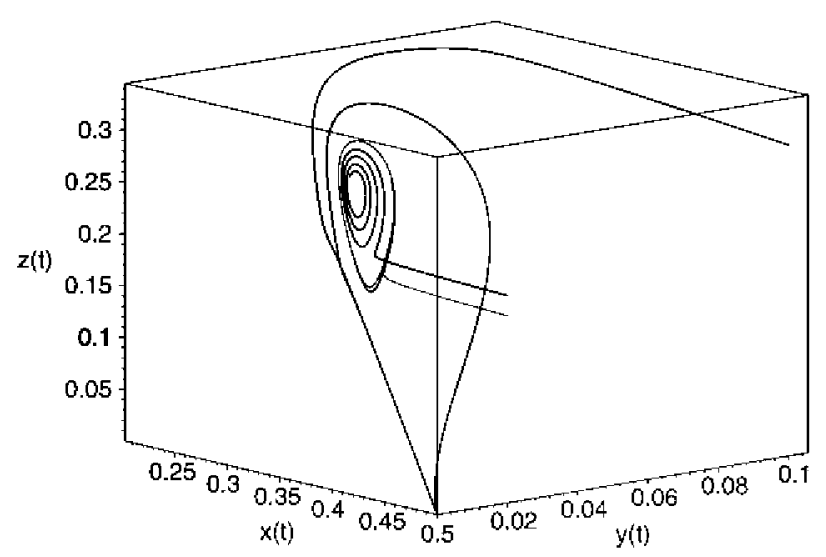

Fig, 8. Bifurcations of stationary values of fixed point components (A tridimensional picture corresponding to Fig. 7). Note that the attractors are placed on a quasi-plane manifold.

analysis of the original model (3)-(5) is considerably simplified in the case of a constant and large owners population, as discussed in Section 2 above. In particular, it is shown therein that the guards population evolution is driven by the number of crimes being committed at any given time. Parameters $f$ and $g$ in (10) and (11) account for the quantitative aspects of this trend. Under general assumptions, an oscillatory dynamic sets in. Roughly speaking, the more the resources are, the larger becomes the population of criminals. This phenomenon induces a rise in the number of security forces available to fight criminals off. Subsequently, criminal numbers diminish and so do the security forces, eventually allowing for an increase in crimes to occur - which leads to a new cycle in this periodic behaviour. These types of dynamics are ubiquitous in biology and ecology

. However, there do not seem to be many examples of non-human societies displaying the particular kind of interspecific predator-prey relations considered in this work.

An issue addressed in Section 2 also is that of the influence of the parameters $H$ and $h$ in the case of competition among criminals and guards. Under a particular form of that assumption, it has been shown that only in the limit case $h=0$ (a total absence of casualties in the security forces) does a suitable increase in $H$ ensure the extinction of criminals under general initial conditions. When $h>0$, however small, a strong depletion of the security forces is then possible.

Finally, we have performed in Section 3 a preliminary study of the complete system (3)-(5). We have reduced ourselves to discussing the bifurcation diagram in terms of parameter $H$ for a given reference set of parameter values (cf. (27)). These results, however partial, show a rich variety of dynamic behaviours as $H$ varies. We consider these examples merely first steps towards a more detailed study of the complete system that we intend to do in the future.

\section{Acknowledgments}

This work has been partially supported by Italy-Spain Integrated Action HI2006-0026 as well as by Acción Especial UCM AE10/07-15449 and the MEC project FIS2006-08607. We also acknowledge gratefully the suggestions made by two anonymous referees.

\section{References}

Hammurabi's code of laws is arguably the oldest penal text. It can be retrieved at the address: http://www.fordham.edu/halsall/ancient/ hamcode.html.

E. Durkheim, Le crime phénomène normal 1894, in: Les regles de la méthode sociologique. Paris 14 ed. 1960, 65-72.

S. Kanazawa, M.C. Still, Why men commit crimes (and why they desist), Sociological Theory 18 (2000) 434-447.

P. Ormerod, Crime, economic incentives and social networks, The Institute of Economic Affairs, London (2005).

L.E. Cohen, M. Felson, Social change and crime rate trends: A routine activity approach, American Sociological Review 44 (1979) 588-608.

L.E. Cohen, R. Machalek, A general theory of expropriative crime: An evolutionary ecological approach, American Journal of Sociology 3 (1988) 465-501.

M. Felson, R.V. Clarke, Opportunity makes the thief. Police research series, 98. Home Office (UK), 1998.

L. Real, The kinetics of functional response, American Naturalist 111 (1977) 289-300. 
A.A. Berryman, The origins and evolution of predator-prey theory, Ecology 73 (1992) 1530-1535.

R.M. May, Stability and Complexity in Model Ecosystems, Princeton University Press, 2001.

O. Bäckman, C. Edling, Mathematics matters: On the absence of mathematical models in quantitative sociology, Acta Sociologica 42 (1999) $69-78$.

P. Ball, The physical modelling of human social systems, ComPlexUs 1 (2004) 190-206.

C.R. Edling, Mathematics in sociology, Annual Review of Sociology 28 (2002) 197-220.

P. Ormerod, C. Mounfield, L. Smith, Non-linear Modelling of Burglary and Violent Crime in the UK. Volterra Consulting Ltd, 2001.

M. Campbell, P. Ormerod, Social interaction and the dynamics of crime, Volterra Consulting Ltd, 1997.

H. Zhao, Z. Feng, C. Castillo-Chávez, The Dynamics of Poverty and Crime, preprint MTBI-02-08M 9, 2002.

D. Usher, The dynastic cycle and the stationary state, American Economic Review 79 (1989) 1031-1044.

G. Feichtinger, C.V. Forst, C. Piccardi, A nonlinear dynamical model for the dynastic cycle, Chaos, Solitons and Fractals 7 (1996) $257-271$.

E. Hairer, G. Wanner, Analysis by its History, Springer, New York, 1995.

Yu.A. Kuznetsov, Elements of Applied Bifurcation Theory, Springer-Verlag, New York, 2004.

S.H. Strogatz, Non-linear Dynamics and Chaos, Perseus book Publishing LLC, USA, 2000.

A. Panfilov, Non-linear Dynamical Systems. 2001. Online publication in: http://www-binf.bio.uu.nl/panfilov/nonlin.html.

J.D. Murray, Mathematical Biology, Springer Verlag, New York, 2002.

S. Camazine, J.-L. Deneubourg, N.R. Franks, J. Sneyd, G. Theraulaz, E. Bonabeau, Self-Organization in Biological Systems, Princeton University Press, New Jersey., 2001.

A. Quetelet, Sur l'homme et le developpement de ses facultes, ou Essai de physique sociale, Bachelier, Paris, 1835.

E.L. Glaeser, B. Sacerdote, J,A. Scheinkman, Crime and Social interactions, The Quarterly Journal of Economics 111 (2) (1996) 507-548.

M. Eigen, J.Mc. Caskill, P. Schuster, The molecular quasispecies, Advances in Chemical Physics 75 (1989) 149-263.

S. Cote (Ed.), Criminological Theories. Bridging the Past to the Future, Sage Publications Inc, 2002. 